\title{
A practical method for dealing with missing Glasgow Coma Scale verbal component scores
}

\author{
Paul M. Brennan, MBBChir, FRCS, PhD, ${ }^{1}$ Gordon D. Murray, MA, PhD, ${ }^{2}$ and \\ Graham M. Teasdale, MBBS, FRCP, FRCS, DSc Hon ${ }^{3}$
}

${ }^{1}$ Translational Neurosurgery, Centre for Clinical Brain Sciences, University of Edinburgh; 2Usher Institute, University of Edinburgh; and ${ }^{3}$ nstitute of Health and Wellbeing, University of Glasgow, United Kingdom

\begin{abstract}
OBJECTIVE The Glasgow Coma Scale (GCS) is used for the assessment of impaired consciousness; however, it is not always possible to test each component, most commonly the verbal component. This affects the derivation of the GCS sum score, which has a role in systems for predicting patient outcome. Imputation of missing scores does not add extra information, but it does allow use of tools for predicting outcome that require complete data. The authors devised a simple and practical tool to employ when verbal component data are missing. They then assessed the tool's utility by application to the GCS-Pupils plus age plus CT findings (GCS-PA CT) prognostic model.
\end{abstract}

METHODS The authors inspected data from the International Mission for Prognosis and Analysis of Clinical Trials in Traumatic Brain Injury (IMPACT) cohort to characterize the frequency of missing verbal scores. The authors identified a single verbal score to impute for each eye and motor combined sum (EM) score from distributions of verbal scores in a published database of 54,069 patients. The effectiveness of the imputed verbal score was assessed using a dataset containing information from the IMPACT and Corticosteroid Randomisation After Significant Head Injury (CRASH) databases. The authors compared the performance of the prognostic model using actual verbal scores with the performance using imputed verbal scores and assessed the information yield using Nagelkerke's $\mathrm{R}^{2}$ statistic.

RESULTS Verbal data were most commonly missing in patients with no eye opening and with a motor score of 4 or less. The "simple" imputation model that was developed performed as well as a more complex model involving distinct combinations of eye and motor scores. The imputation model consisted of the following: EM scores 2-6, add 1; EM score 7, add 2; EM score 8 or 9, add 4; and EM score 10, add 5 to provide the GCS sum score. Modeling without information about the verbal score reduced the $\mathrm{R}^{2}$ from $32.1 \%$ to $31.4 \%$ and from $34.9 \%$ to $34.0 \%$ for predictions of death and favorable outcome at 6 months, respectively, compared with using full verbal score information.

CONCLUSIONS This strategy is particularly valuable for imputation in clinical practice, enabling clinicians to make a rapid and reliable determination of the GCS sum score when the verbal component is not testable. This will support clinical communication and decisions based on estimates of injury severity as well as enable estimation of prognosis. The authors suggest that external validation of their imputation strategy and the performance of the GCS-PA charts should be undertaken in other clinical populations.

https://thejns.org/doi/abs/10.3171/2020.6.JNS20992

KEYWORDS Glasgow Coma Scale; GCS; head injury; missing data; verbal score; traumatic brain injury

$\mathrm{T}$ HE Glasgow Coma Scale (GCS) has been widely adopted for the assessment of impaired consciousness in patients with a head injury or other types of acute brain damage. ${ }^{1}$ Its findings support clinical decisionmaking, communication, and assessment of severity of brain injury in the acute stage as well as likely outcome. ${ }^{2,3}$ However, for practical reasons, it is not always possible to test each of the three components of the scale. None can be validly assessed in a pharmacologically sedated and paralyzed patient. Loss of a single component occurs most often with the verbal component ${ }^{4}$ usually as a result of intubation. This can be documented in clinical practice as "not testable" and can be compensated for by using motor and eye findings to guide the care of the patient. ${ }^{5}$ However,

ABBREVIATIONS CRASH = Corticosteroid Randomisation After Significant Head Injury; EM = eye and motor combined sum; E1-4 = eye scores; GCS = Glasgow Coma Scale; GCS-P = GCS-Pupils; GCS-PA = GCS-Pupils plus age; GCS-PA CT = GCS-Pupils plus age plus CT findings; IMPACT = International Mission for Prognosis and Analysis of Clinical Trials in Traumatic Brain Injury; M1-6 = motor scores; TARN = Trauma Audit and Research Network; TBI = traumatic brain injury; VSTR = Victorian State Trauma Registry; $\mathrm{V} 1-5$ = verbal scores.

SUBMITTED March 29, 2020. ACCEPTED June 11, 2020.

INCLUDE WHEN CITING Published online September 8, 2020; DOI: 10.3171/2020.6.JNS20992. 
an inability to test one of the components of the GCS affects the derivation of its sum score.

The GCS sum score is used clinically as a "shorthand" device to summarize and communicate a patient's condition and to stratify or grade this condition as a guide to case management. It is also widely used as a component of systems to rate the severity of acute illnesses or injury, such as the Acute Physiology and Chronic Health Evaluation II (APACHE II), ${ }^{6}$ Revised Trauma Score, ${ }^{7}$ Trauma and Injury Severity Score (TRISS), ${ }^{8}$ Simplified Acute Physiology Score (SAPS), ${ }^{9}$ A Severity Characterization of Trauma (ASCOT), ${ }^{10}$ and World Federation of Neurosurgical Societies' Subarachnoid Hemorrhage Scale. ${ }^{11}$ The GCS sum score also has a key place in formal multivariate systems for predicting outcome. These range in complexity from the GCS-Pupils (GCS-P) score, ${ }^{3}$ a simple, quick arithmetic calculation, to more complex multivariate models, such as the International Mission for Prognosis and Analysis of Clinical Trials in Traumatic Brain Injury (IMPACT). ${ }^{12}$

A number of imputation methods for dealing with missing GCS data have been proposed, although alternative approaches to imputation can result in different predictions of mortality. ${ }^{13-16}$ These imputation strategies are principally used to support the analysis of trauma registry data, in which missing GCS observations can be as high as $20 \%^{13}$ and are most commonly associated with more severely injured patients. ${ }^{17}$ Imputation of missing scores does not add any extra information; it simply allows the use of tools that require the data to be complete.

The assignment of a score of 1 to untestable components does not provide a valid index of the patient's condition and can misleadingly overestimate its severity. ${ }^{5}$ An abridged score based on measurements of only the testable features is similarly deficient. Proposed methods that use an association between findings in the eye, motor, and verbal components of the GCS to mathematically model the missing verbal component ${ }^{18-20}$ may have no advantage over other imputation strategies and are unlikely to improve outcome predication..$^{19,21}$ In summary, the imputation methods described to date may have a role in research, but they are not practical to implement in the clinic.

The aims in this study were to investigate the frequency of the loss of verbal score in head injuries of different severities; establish the pattern of relationships between eye, motor, and verbal scores; and use the findings as a basis for devising a simple, practical, and reliable tool to employ when verbal component data are missing. Finally, the utility of this approach was assessed by application to the GCS-Pupils plus age plus CT findings (GCS-PA CT) prognostic model that we described recently. ${ }^{22}$ This model enables graphical display of probabilities of outcome in a simple, easy-to-use format that supports and promotes the incorporation of prognostic information in patient care.

\section{Methods}

\section{"Missingness": Frequency and Pattern}

Investigations into the frequency and pattern of missing data were conducted using the IMPACT database. ${ }^{12}$ The IMPACT core database contains data from 11,989 patients with traumatic brain injury (TBI) that were collected prospectively in 11 different studies including eight randomized controlled trials and three epidemiological studies. We inspected data from the IMPACT cohort to characterize the frequency of missing verbal scores. Using data from all 11,989 patients, all available baseline assessments of the GCS were taken by pooling prehospital, first-hospital, admission-to-study hospital, and postresuscitation GCS scores.

The distribution of verbal scores for each eye and motor score combination was then determined on the basis of a publication that combined data from three different registries-Trauma Audit and Research Network (TARN), Victorian State Trauma Registry (VSTR), and Corticosteroid Randomisation After Significant Head Injury (CRASH) trial data - to create a larger database than IMPACT; this larger database included 54,069 patients. ${ }^{23}$ From this we could identify an appropriate verbal score to impute for each eye and motor score combination.

\section{Effect of Imputation on Information Content}

To assess the effectiveness of the imputed verbal score value determined from the aforementioned analysis of 54,069 patients, we used a dataset containing pooled information from the IMPACT and $\mathrm{CRASH}^{24}$ databases, which we previously described in the derivation of the GCS-P score and GCS-PA CT prognostic charts. ${ }^{3,22}$ Patients were identified for whom GCS, concurrent pupil reactivity, age, and CT imaging findings data were available. The proportions of patients who either were dead or had an unfavorable outcome at 6 months (vegetative state or severe disability) according to the Glasgow Outcome Scale were determined. We compared the performance of prognostic models when using actual verbal scores with the performance of models when using imputed verbal scores, according to our strategy described below, and assessed the information yield using Nagelkerke's $\mathrm{R}^{2}$ statistic. ${ }^{25}$

\section{Results}

\section{Frequency and Pattern of "Missingness"}

From our analysis of the IMPACT data, there were 11,989 patients in which both motor and eye scores were available. We determined how often the GCS verbal score was missing for each combination of eye and motor score (Table 1). Verbal data were most commonly missing in patients with no eye opening (eye score 1 or E1 [73.6\% of total patient assessments]) and in patients with a motor score of 4 or less (76.8\% of the E1 cohort). The higher the eye and motor scores, the less likely it was that the verbal score would be missing; for example, in patients with a motor score of 6 , only $2 \%$ of verbal data were missing. Overall, the verbal score was missing for $11 \%$ of the assessments.

We next examined the distribution of GCS verbal scores, according to combinations of eye and motor scores, in a published dataset that combined the TARN, VSTR, and CRASH databases. The specificity of a single verbal score for imputation for any given eye and motor score combination was greatest for low eye and motor scores (Table 2). 
TABLE 1. Percentage of assessments with a missing GCS verbal score for each combination of GCS eye and motor scores

\begin{tabular}{cccccc}
\hline Motor & \multicolumn{5}{c}{ Eye Score } \\
\cline { 2 - 6 } Score & E1 & E2 & E3 & E4 & All \\
\hline M1 & $18.6(3421)$ & $12.8(39)$ & $15.0(20)$ & $10.3(29)$ & $18.5(3509)$ \\
\hline M2 & $12.9(2467)$ & $12.2(74)$ & $25.0(12)$ & $5.0(20)$ & $12.9(2573)$ \\
\hline M3 & $12.5(2563)$ & $5.2(192)$ & $8.3(48)$ & $2.8(36)$ & $11.8(2839)$ \\
\hline M4 & $12.7(4198)$ & $5.3(604)$ & $6.6(167)$ & $6.4(218)$ & $11.4(5187)$ \\
\hline M5 & $10.4(3713)$ & $7.4(1007)$ & $4.9(691)$ & $6.1(577)$ & $8.8(5988)$ \\
\hline M6 & $3.7(108)$ & $6.5(124)$ & $1.9(537)$ & $1.2(1502)$ & $1.8(2271)$ \\
\hline All & $13.4(16,470)$ & $6.8(2040)$ & $4.4(1475)$ & $3.0(2382)$ & $11.1(22,367)$ \\
\hline
\end{tabular}

Assessments made in 11,989 patients were identified in the IMPACT database. Values are expressed as the percentage of the missing verbal scores for each combination of eye score and motor score (total number of assessments for that combination). Each individual patient could contribute up to four GCS assessments used in this analysis.

The summation of eye and motor scores into an EM score (eye and motor combined sum score) made the selection of what verbal score to impute clearer (Table 3). The specificity of the modal value for each EM combination is highest for EM score 2 (94.2\%); this specificity decreases

TABLE 2. Distribution of verbal scores for given eye score and motor score combinations

\begin{tabular}{|c|c|c|c|c|c|c|}
\hline \multirow{2}{*}{$\begin{array}{l}\text { Motor } \\
\text { Score }\end{array}$} & \multirow{2}{*}{$\begin{array}{l}\text { Eye } \\
\text { Score }\end{array}$} & \multicolumn{5}{|c|}{ Verbal Score } \\
\hline & & V1 & V2 & V3 & V4 & V5 \\
\hline \multirow{4}{*}{ M1 } & E1 & 6847 & 326 & 32 & 40 & 24 \\
\hline & E2 & 71 & 41 & & & \\
\hline & E3 & 73 & 15 & & & \\
\hline & E4 & 107 & 24 & & & \\
\hline \multirow{4}{*}{ M2 } & E1 & 1060 & 233 & 24 & 20 & 26 \\
\hline & E2 & 63 & 87 & & & \\
\hline & E3 & & & & & 25 \\
\hline & E4 & 28 & & & & \\
\hline \multirow{4}{*}{ M3 } & E1 & 969 & 381 & 31 & & \\
\hline & E2 & 110 & 134 & 62 & & \\
\hline & E3 & 30 & 55 & 47 & 24 & \\
\hline & E4 & 44 & 30 & & 29 & \\
\hline \multirow{4}{*}{ M4 } & E1 & 1042 & 517 & 51 & 32 & \\
\hline & E2 & 177 & 310 & 149 & 75 & \\
\hline & E3 & 79 & 125 & 130 & 117 & 33 \\
\hline & E4 & 90 & 126 & 70 & 138 & 72 \\
\hline \multirow{4}{*}{ M5 } & E1 & 1339 & 927 & 191 & 111 & \\
\hline & E2 & 377 & 683 & 501 & 325 & \\
\hline & E3 & 241 & 452 & 622 & 1250 & 175 \\
\hline & E4 & 335 & 417 & 424 & 990 & 342 \\
\hline \multirow{4}{*}{ M6 } & $\mathrm{E} 1$ & 68 & 77 & 32 & 69 & 36 \\
\hline & E2 & 31 & 56 & 76 & 164 & 55 \\
\hline & E3 & 144 & 217 & 385 & 3456 & 2265 \\
\hline & E4 & 223 & 211 & 505 & 6961 & 14,431 \\
\hline
\end{tabular}

Based on data from the TARN, VSTR, and CRASH databases. The modal verbal score in each row is presented in boldface type. as the EM score increases to 7 (EM scores and associated specificities: $3,76.7 \%$; 4, 68.3\%; 5, 59.8\%; 6, 44.5\%; and $7,33.2 \%$, respectively) before increasing again (EM scores and associated specificities: 8, 42.9\%; 9, 49.5\%; and 10, $64.6 \%$, respectively).

The mode verbal score is 1 for EM scores 2-6 (Tables 2 and 3), which corresponds to GCS sum scores 3-7, which are defined as severe brain injury. This includes the range of motor and eye scores for which verbal scores were most commonly missing (Table 1). The appropriate imputed (mode) verbal score value is less clear in the range of EM scores 7-9, although in this cohort, the average proportion of GCS assessments with a missing verbal score was only $5.1 \%$ (Table 1). For no EM score combination was the imputed verbal score 3 .

\section{Information Gained From Imputation}

Using the imputed verbal scores, based on both eye and motor scores and also on the EM score, we assessed how the information provided about outcome from the GCS-PA CT prognostic charts was affected by the imputation (Table 4). We examined the effect of substituting the imputed verbal score for the actual verbal score in a model using

TABLE 3. Distribution of verbal scores for given EM scores

\begin{tabular}{|c|c|c|c|c|c|c|}
\hline \multirow{2}{*}{$\begin{array}{c}\text { EM } \\
\text { Score }\end{array}$} & \multicolumn{5}{|c|}{ Verbal Score } & \multirow{2}{*}{$\begin{array}{l}\% \text { Total of } \\
\text { Mode Score }\end{array}$} \\
\hline & V1 & V2 & V3 & V4 & V5 & \\
\hline 2 & 6847 & 326 & 32 & 40 & 24 & 94.2 \\
\hline 3 & 1131 & 274 & 24 & 20 & 26 & 76.7 \\
\hline 4 & 1105 & 483 & 31 & & & 68.3 \\
\hline 5 & 1259 & 675 & 113 & 32 & 25 & 59.8 \\
\hline 6 & 1514 & 1292 & 387 & 210 & & 44.5 \\
\hline 7 & 568 & 915 & 663 & 540 & 69 & 33.2 \\
\hline 8 & 362 & 634 & 768 & 1552 & 302 & 42.9 \\
\hline 9 & 479 & 634 & 809 & 4446 & 2607 & 49.5 \\
\hline 10 & 223 & 211 & 505 & 6961 & 14,431 & 64.6 \\
\hline
\end{tabular}

Based on data obtained from the TARN, VSTR, and CRASH databases. Modal values for imputation are presented in boldface type. 
TABLE 4. Assessment of $R^{2}$ values for models predicting death and favorable outcome based on the GCS score, pupil reactivity, patient age, and CT abnormality count, using actual and imputed verbal scores

\begin{tabular}{lcc}
\hline & $\begin{array}{c}\text { Predicting } \\
\text { Death (\%) }\end{array}$ & $\begin{array}{c}\text { Predicting Favorable } \\
\text { Outcome (\%) }\end{array}$ \\
\hline Actual verbal score & 32.1 & 34.9 \\
\hline Simple imputation & 31.4 & 34.0 \\
\hline Complex imputation & 31.3 & 34.1 \\
\hline
\end{tabular}

The simple model is based on the EM score, and the complex model uses distinct combinations of eye and motor scores.

the GCS, pupil reactivity, age, and CT abnormality count. This analysis relied on data from the pooled IMPACT and CRASH databases that were used for the original derivation of the GCS-PA CT prognostic charts.,22

The proportion of variability in outcome "explained" by this approach was assessed using Nagelkerke's $\mathrm{R}^{2}$ statistic. The "simple" imputation determined by selecting the verbal score according to the EM score performed as well as the more complex model using distinct combinations of eye and motor scores. Modeling without information about the verbal score reduced the $\mathrm{R}^{2}$ from $32.1 \%$ to $31.4 \%$ and from $34.9 \%$ to $34.0 \%$ for predictions of death and favorable outcome at 6 months, respectively, compared with using full verbal score information (Table 4).

\section{Aid to Imputation}

Based on the imputed verbal score for each EM score, we have developed a simple visual aid to support imputation of a missing verbal score in the clinic in order to facilitate determination of the GCS-P score and predictions of outcomes using the GCS-PA prognostic charts (Fig. 1). In step 1, the eye and motor component scores are summed. In step 2 , the verbal component score is identified and added to the EM score, giving an imputed GCS sum score in step 3 .

\section{Discussion}

Our findings show that, based on the large IMPACT database, overall all three components of the GCS could be determined in almost $90 \%$ of prehospital, first-hospital, admission-to-study hospital, or post-resuscitation GCS assessments. Among assessments in which both eye and motor components were recorded, only $11 \%$ did not have a verbal score. The verbal score was most often missing in patients with a severe head injury, characterized by no eye opening and not obeying commands (a combined EM score of 6 or less); these patients are usually intubated. The verbal score was rarely missing in the remaining assessments of patients with a mild or moderate head injury, who in practice make up the great majority of admitted head injuries.

Our analysis of associations among the three components of the scale demonstrates that if the verbal response cannot be assessed, a simple and accurate strategy for imputing missing GCS verbal score data is possible, based on a combination of eye and motor scores. The pattern of associations among the three GCS components showed that for any combined eye and motor (EM) score of 6 or below, the verbal score was most frequently 1 . For EM scores 7 and 8 , the most common verbal scores were 2 and 4, respectively, but these were seen in fewer than onehalf of the patients. For EM scores 9 and 10, verbal scores of 4 and 5, respectively, were recorded for one-half and two-thirds of patients, respectively. These data support a simple pragmatic strategy for imputing a missing verbal score from the modal value for each combined EM score, as illustrated in Fig. 1.

The appropriate verbal score to impute is unambiguous in severely or mildly head-injured patients, but it is less distinct in moderately head-injured patients. Interestingly, a verbal score of 3 (or V3) was not imputed from any EM combination. This probably reflects our observation that the V3 verbal response was least often recorded in these data and may indicate uncertainty in how the V3 score (intelligible single words) is differentiated in clinical assessment from the V2 (only moans/groans) and V4 (not orientated but communicates coherently) scores.

A consequence of our verbal score imputation strategy is that several possible GCS sum (EVM) score combinations are absent in the visual aid to imputation. The lack of a sum score of 8 is of particular interest, because of its long-established role in defining a severe, as opposed to a moderate, injury. GCS score 8 is customarily equated with a combined score of E1 plus V2 plus M5 (motor score of 5 ). In the visual score imputation system that we have proposed, an EM score of 6 can be taken to define severe injury. Classification of a patient as having a moderate brain injury (GCS scores 9-12) rather than a mild brain injury (GCS scores 13-15), based on the imputed verbal score, will most likely result from an assessment of the eye score as E2 rather than E3. Treatment decisions should be made from the integration of all available clinical and prognos-

\begin{tabular}{|c|c|c|c|c|c|c|c|c|c|c|}
\hline Step 1 & $E+M$ & 2 & 3 & 4 & 5 & 6 & 7 & 8 & 9 & 10 \\
\hline Step 2 & $+V$ & \multicolumn{5}{|c|}{ Add 1} & 2 & \multicolumn{2}{|c|}{4} & 5 \\
\hline Step 3 & EVM & 3 & 4 & 5 & 6 & 7 & 9 & 12 & 13 & 15 \\
\hline
\end{tabular}

FIG. 1. Visual aid to imputation of a verbal score based on the EM score. In step 1, the eye and motor component scores are summed. In step 2, the verbal component score is identified and added to the EM sum score, giving an imputed GCS sum (EVM) score in step 3. Figure is available in color online only. 
tic data, and any change in this information over time, not on the basis of any single score or classification.

We assessed the merit of imputation of a verbal score by analyzing the extent of outcome information modeled using age, total GCS score, pupil reaction, and CT scan findings, as described previously. ${ }^{22}$ The information yielded by inputting missing verbal information using our simple imputation method is closely comparable to that obtained by estimating a score based on precise eye and motor findings and close to that obtained from full information about the three components of the total GCS score. Our simple imputation approach may obviate the need for more complex imputation approaches when a sum score is needed. ${ }^{13-16,18,20}$

The purpose of our imputation strategy, to permit the sum score to be determined easily for an individual patient in clinical practice, contrasts with that of existing imputation strategies. Those models were developed to support data registries and calculation of prognostic tools, such as the Trauma Score or APACHE scoring systems..$^{13,15,18,20}$ Their complexity is not suited to the clinic. Direct comparison of our imputation strategy with existing models is therefore not helpful. Further, those models were developed using linear regression approaches, limiting the possibility to directly compare performance to our model, which was built using logistic regression modeling.

A strength of this study is the large number of patient GCS observations that have been used, from several different, carefully collected, and well-characterized databases of head-injured patients. Nevertheless, although the merit of simple verbal imputation is clear in principle, calibration of the findings is very data intensive. Additional observations in other large, contemporary population-based registries of head-injured patients will be useful in finetuning the detail and precision of the proposed system for imputation.

We do not propose that imputed verbal scores should be included in clinical charts. The principle of using the GCS for assessment of coma and impaired consciousness is to record what is actually observed. The utility of serial assessment and recording of actual observations to assess for changes in level of consciousness is not necessarily precluded by missing data for one response. An imputed GCS sum score should not be used as a baseline from which to decide if a subsequent full assessment represents a change requiring action. The GCS score that is communicated should be actual and not imputed.

The use of imputed verbal scoring may be best reserved for providing a GCS sum score to enable the calculation of severity and prognostic scoring systems such as the GCS-P score and assessment of a patient's predicted outcome at 6 months using the GCS-PA prognostic charts, which we described recently., ${ }^{3,2}$ The GCS-P and GCS-PA charts are simple arithmetic scores that extend the information provided about patient outcome to an extent comparable to that obtained using more complex methods.,22

Imputation of missing scores does not add any extra information; it simply allows the use of tools that require the data to be complete. The GCS-PA charts provide a rapid assessment of a patient's predicted outcome at 6 months, which can support clinician decision-making and com- munication between clinical teams. Although clinicians identify prognosis as a factor in their decision-making, the prognosis remains exposed to personal subjective opinions. ${ }^{26,27}$ Estimates of prognosis are best made using mathematical methods that combine information about multiple aspects of the patient's condition, but such multivariate models have not found widespread acceptance in clinical practice, ${ }^{28}$ leading to errors in case management. The GCS-PA prognostic charts are presented in a userfriendly way so that they can easily be applied in the clinical scenario and be clinically useful.

\section{Conclusions}

Our imputation strategy is particularly valuable for imputation of missing verbal score data in clinical practice. Verbal score imputation will enable clinicians to make a rapid and reliable determination of the GCS sum score in cases in which the verbal component is not testable. This will support communication between clinicians and clinician decision-making based on estimates of severity of brain injury and will enable estimation of prognosis using GCS-P scores and GCS-PA prognostic charts. External evaluation of our imputation strategy and the performance of GCS-PA charts should be undertaken in other clinical populations. ${ }^{29}$

\section{References}

1. Teasdale G, Maas A, Lecky F, et al. The Glasgow Coma Scale at 40 years: standing the test of time. Lancet Neurol. 2014;13(8):844-854.

2. Jennett B, Teasdale G, Braakman R, et al. Predicting outcome in individual patients after severe head injury. Lancet. 1976;1(7968):1031-1034.

3. Brennan PM, Murray GD, Teasdale GM. Simplifying the use of prognostic information in traumatic brain injury. Part 1: The GCS-Pupils score: an extended index of clinical severity. J Neurosurg. 2018;128(6):1612-1620.

4. Healey C, Osler TM, Rogers FB, et al. Improving the Glasgow Coma Scale score: motor score alone is a better predictor. J Trauma. 2003;54(4):671-680.

5. Teasdale G, Allan D, Brennan P, et al. Forty years on: updating the Glasgow Coma Scale. Nurs Times. 2014;110(42): $12-16$.

6. Knaus WA, Draper EA, Wagner DP, Zimmerman JE. APACHE II: a severity of disease classification system. Crit Care Med. 1985;13(10):818-829.

7. Champion HR, Sacco WJ, Copes WS, et al. A revision of the trauma score. J Trauma. 1989;29(5):623-629.

8. Boyd CR, Tolson MA, Copes WS. Evaluating trauma care: the TRISS method. Trauma Score and the Injury Severity Score. J Trauma. 1987;27(4):370-378.

9. Le Gall JR, Loirat P, Alperovitch A, et al. A simplified acute physiology score for ICU patients. Crit Care Med. 1984; 12(11):975-977.

10. Champion HR, Copes WS, Sacco WJ, et al. A new characterization of injury severity. J Trauma. 1990;30(5):539-546.

11. Teasdale GM, Drake CG, Hunt W, et al. A universal subarachnoid hemorrhage scale: report of a committee of the World Federation of Neurosurgical Societies. Letter. J Neurol Neurosurg Psychiatry. 1988;51(11):1457.

12. Marmarou A, Lu J, Butcher I, et al. IMPACT database of traumatic brain injury: design and description. J Neurotrauma. 2007;24(2):239-250. 
13. Moore L, Lavoie A, LeSage N, et al. Multiple imputation of the Glasgow coma score. J Trauma. 2005;59(3):698-704.

14. Moore L, Hanley JA, Turgeon AF, et al. A multiple imputation model for imputing missing physiologic data in the National Trauma Data Bank. J Am Coll Surg. 2009;209(5): 572-579.

15. Moore L, Hanley JA, Lavoie A, Turgeon A. Evaluating the validity of multiple imputation for missing physiological data in the National Trauma Data Bank. J Emerg Trauma Shock. 2009;2(2):73-79.

16. Glance LG, Osler TM, Mukamel DB, et al. Impact of statistical approaches for handling missing data on trauma center quality. Ann Surg. 2009;249(1):143-148.

17. O'Reilly GM, Cameron PA, Jolley DJ. Which patients have missing data? An analysis of missingness in a trauma registry. Injury. 2012;43(11):1917-1923.

18. Meredith W, Rutledge R, Fakhry SM, et al. The conundrum of the Glasgow Coma Scale in intubated patients: a linear regression prediction of the Glasgow verbal score from the Glasgow eye and motor scores. J Trauma. 1998;44(5):839845.

19. Hannan EL, Farrell LS, Bessey PQ, et al. Accounting for intubation status in predicting mortality for victims of motor vehicle crashes. J Trauma. 2000;48(1):76-81.

20. Rutledge R, Lentz CW, Fakhry S, Hunt J. Appropriate use of the Glasgow Coma Scale in intubated patients: a linear regression prediction of the Glasgow verbal score from the Glasgow eye and motor scores. J Trauma. 1996;41(3):514522.

21. Cheng K, Bassil R, Carandang R, et al. The estimated verbal GCS subscore in intubated traumatic brain injury patients: Is it really better? J Neurotrauma. 2017;34(8):1603-1609.

22. Murray GD, Brennan PM, Teasdale GM. Simplifying the use of prognostic information in traumatic brain injury. Part 2: Graphical presentation of probabilities. J Neurosurg. 2018; 128(6):1621-1634.

23. Reith FCM, Lingsma HF, Gabbe BJ, et al. Differential effects of the Glasgow Coma Scale score and its components: an analysis of 54,069 patients with traumatic brain injury. Injury. 2017;48(9):1932-1943.
24. Roberts I, Yates D, Sandercock P, et al. Effect of intravenous corticosteroids on death within 14 days in 10008 adults with clinically significant head injury (MRC CRASH trial): randomised placebo-controlled trial. Lancet. 2004;364(9442): $1321-1328$

25. Nagelkerke NJD. A note on a general definition of the coefficient of determination. Biometrika. 1991;78(3):691-692.

26. Barlow P, Teasdale G. Prediction of outcome and the management of severe head injuries: the attitudes of neurosurgeons. Neurosurgery. 1986;19(6):989-991.

27. Moore NA, Brennan PM, Baillie JK. Wide variation and systematic bias in expert clinicians' perceptions of prognosis following brain injury. Br J Neurosurg. 2013;27(3):340-343.

28. Steyerberg EW, Moons KGM, van der Windt DA, et al. Prognosis Research Strategy (PROGRESS) 3: prognostic model research. PLoS Med. 2013;10(2):e1001381.

29. Kappen TH, van Klei WA, van Wolfswinkel L, et al. Evaluating the impact of prediction models: lessons learned, challenges, and recommendations. Diagn Progn Res. 2018;2(1):11.

\section{Disclosures}

The authors report support from a Muriel Cooke bequest to the University of Glasgow for incidental costs.

\section{Author Contributions}

Conception and design: Brennan, Teasdale. Acquisition of data: all authors. Analysis and interpretation of data: all authors.

Drafting the article: all authors. Critically revising the article: all authors. Reviewed submitted version of manuscript: all authors. Approved the final version of the manuscript on behalf of all authors: Brennan. Statistical analysis: Murray.

\section{Correspondence}

Paul M. Brennan: Translational Neurosurgery, Centre for Clinical Brain Sciences, University of Edinburgh, United Kingdom. paul. brennan@ed.ac.uk. 